Living guidelines: using living systematic reviews to support up-to-date practice guidelines

Authors: Elie A. Akl, Joerg J Meerpohl, Julian Elliott, Lara Kahale, Holger J. Schünemann on behalf of the Living Systematic Review Network

Word Count: 2903 words

Corresponding author:

Elie A. Akl, MD, MPH, PhD

Department of Internal Medicine

American University of Beirut Medical Center, P.O. Box: 11-0236, Riad-El-Solh Beirut 1107, 2020

Beirut, Lebanon

Email: ea32@aub.edu.lb 


\begin{abstract}
While it is important for the evidence supporting practice guidelines to be current, that is often not the case. The advent of living systematic reviews has made the concept of "Living Guidelines" realistic, with the promise to provide timely, up-to-date and high quality guidance to target users. However, achieving living guidelines requires specific methodology for their establishment, continual development, and dissemination. We define living guidelines as an optimization of the guideline development process to allow updating individual recommendations as soon as new relevant evidence becomes available. It then discusses when living guidelines are appropriate, the workflows required to support them, the collaboration between LSR and living guideline teams, the thresholds for changing recommendations, and potential approaches to publication and dissemination. The success and sustainability of the concept of living guideline will depend on those of its major pillar, the living systematic review. We conclude that guideline developers should both experiment with and research the process of living guidelines.
\end{abstract}




\section{What is new}

- Living guidelines aim to provide timely advice for decision makers by optimizing the guideline development process to allow updating individual recommendations as soon as new relevant evidence becomes available.

- Guideline developers need to prioritize the recommendations to switch the living status, according to explicit criteria

- Implementing living guidelines will require both workflows to support them, and close collaboration between LSR and living guideline teams

- Challenges that will face the implementation of living guidelines include setting the thresholds for changing recommendations, and potential approaches to publication and dissemination

- The success and sustainability of the concept of living guideline will depend on those of its major pillar, the living systematic reviews 


\section{Introduction}

The Institute of Medicine defines clinical practice guidelines as "statements that include recommendations intended to optimize patient care that are informed by a systematic review of evidence and an assessment of the benefits and harms of alternative care options".(1) Guidelines are intended to support clinicians and their patients in making health-related choices to optimize health outcomes.(1)

A typical guideline includes several recommendations, each addressing alternative management options in a specific population. Two of the main factors determining the recommendation's strength (i.e., strong, conditional) and direction (i.e., in favor or against) are the balance of benefits and harms and the quality of supporting evidence. $(2,3)$ These two factors are judged based on a systematic review of the most current evidence on the relative benefits and harms of the alternative management options under consideration.(4)

A recommendation is as up-to-date as the search date of the supporting systematic review. One study conducted a survival analysis of systematic reviews, i.e., assessed the period over which they remain up-to-date. (5) It found that the median "survival" of reviews in an area with a relatively high rate of publication (i.e., cardiovascular disease) was 2.9 years $(95 \% \mathrm{Cl}$ 1.1-5.3).(5) Moreover, some reviews were already out-of-date by the time of their publication.(5) Given a guideline is based on a set of systematic reviews, some of its recommendations would be out-of-date by the time of the guideline publication. This problem is accentuated when factoring in the additional amount of time needed to move from the evidence to the recommendations and to publish the guidelines. Also, an increasing number of recommendations would become out-of-date as time elapses post guideline publication. Indeed, a study of clinical guidelines developed in the Spanish National Health System found that a fifth of recommendations were out-of-date at three years post publication.(6) 
The most common model of updating recommendations is to update the entire guideline at specific time intervals (e.g., at 3 years) following its publication. This approach has two major limitations. First, some of the recommendations will be out-of-date for varying periods of time (directly correlated to the supporting systematic reviews becoming out-ofdate). This negatively affects the validity of these recommendations. The second limitation is that for some recommendations, efforts invested in their update will be wasted as the underlying evidence will not change. This negatively affects the efficiency of the process.

Conducting more frequent updates of the guideline will enhance validity but negatively impact efficiency. Conducting less frequent updates will enhance efficiency but negatively impact validity. We propose living guidelines as a process to address this challenge. We hypothesize that this process will lead to more valid recommendations, while potentially improving efficiency of guideline development. Although guideline developers have been discussing the concept of living guidelines,(7-9) a systematic review of methodological handbooks did not identify specific instructions for conducting them.(10)

This is the fourth and last paper in a series of papers discussing the emerging field of living systematic reviews. In this paper, we define living guidelines and discuss when they are appropriate, the workflows required to support them, the collaboration between LSR and living guideline teams, the thresholds for changing recommendations, and potential approaches to publication and dissemination.

\section{Definition of living guidelines}

We define a living guideline as an optimization of the guideline development process to allow updating of individual recommendations as soon as new relevant evidence becomes available. The ultimate aim is to provide timely and trustworthy advice for decision makers. One implication of this definition is that the living guideline should build on an existing, high quality guideline developed using the standard process. The second implication is that the 
unit of update becomes the recommendation, and not the guideline. In other terms, the different recommendations included in the guideline will get updated at different times.

\section{When are living guidelines appropriate?}

Adopting a living guideline process will require the prioritization of recommendations to switch to the living status, in order to maximize the value of the approach. The prioritization criteria for living recommendations resemble those proposed by Elliott et al. for prioritizing living systematic review,(JCExx) and include:

1. The recommendation is a priority for the users of the guidelines. This could be affected by a high prevalence of the condition, high rates of associated morbidity and mortality, known variation in practice, and interest in emerging interventions and diagnostic tools.

2. There is a reasonable chance that the existing recommendation changes with the emergence of new evidence. This is the case when the strength of the existing recommendation is conditional (as opposed to being strong) due to a very low or low certainty of evidence. This is a scenario where the consideration of emerging evidence may increase the certainty of evidence, subsequently enhancing the strength of the recommendation.

3. Active research addressing the recommendation of interest is ongoing with a reasonable likelihood of findings being published over the period when the living guideline process is implemented.

At the same time, it is important to keep in mind that recommendations in the same guidelines might not be totally independent from each other. For example, the recommendation to treat with option A might depend on the recommendation to apply test $X$. If the recommendation to apply test $X$ is changed, this might impact on the treatment recommendation. So, the interdependency of these two recommendations would require both to be living recommendations to ensure simultaneous updating. 
When at least one of the above listed criteria for switching a recommendation into a living status cease to apply, the authors could switch back from the living guideline process to the standard guideline process, for individual recommendations or entire guidelines.

\section{Workflows required to support living guideline}

Several guideline handbooks and publications have proposed different approaches for the guideline development and update processes. A review of these proposals found that they overlap to a large extent and proposed a checklist (the Guidelines Development Checklist) of 18 topics and 146 items.(4) Living guidelines do not represent a new approach, but rather an optimization of the standard process (e.g., following the Guidelines Development Checklist) to allow updating of individual recommendations as soon as new relevant evidence becomes available. This optimization can be achieved by integrating the following in the standard guideline process:

1. Living systematic review: this is the most essential requirement for a living guideline process. As defined in the first paper of this series, (JCE xx) a LSR is a process that uses continual surveillance of the literature to allow updating of a systematic review with new evidence as it becomes available.

2. 'Living summary tables': guideline panels rely on standardized summary tables to make the judgments required for developing recommendations. The first type of tables is the Evidence Profile, which provides for each outcome of interest the statistical information on the effects on health benefits and harms of the alternative interventions and the detailed assessment of the certainty of supporting evidence. The second type of table is the Evidence to Decision (EtD) Table, which provides for each recommendation the information on the factors needed to judge the strength and direction of that recommendation. These factors include the health effects of interventions, the certainty of evidence, resource use, impact on equity, and 
acceptability of the intervention, among others. Updating these tables as soon as new evidence emerges is needed to swiftly relay the findings of the LSR to guideline panels in a form that allows them to reconsider the recommendation. Hence, the suggestion of using the term 'living summary tables'.

3. 'Living guideline panel' (or 'on-call guideline panel'): typically, recruiting a guideline panel is time consuming in terms of establishing contact, agreeing on the terms of reference, collecting and managing conflicts of interest information and training. Also, convening a panel meeting requires time due to scheduling challenges. A living guideline process can circumvent those challenges by recruiting ahead of time panel members committed to making themselves available within a very short notice, whenever the updating process is triggered. This implies that virtual meetings would be more feasible than in person meetings. Also, a living guideline panel would likely be engaged for more frequent but shorter periods of times, compared with a standard guideline panel. It is also likely that the membership of the panel will change over time. This raises the challenge of preserving the 'institutional memory' of the group. Also, it necessitates a plan for the training of newly recruited members in guideline development methodology.

4. 'Living peer review process': similarly to recruiting panelists, recruiting peer reviewers may be time consuming and their response may not be timely. A living guideline process could recruit a larger number of reviewers than needed and ensure their commitment to a timely review. The guideline developer could minimize the involvement of the reviewers and provide them with advance notice (e.g., as soon as the updating process is triggered). Also, any internal (e.g., professional society's) or external (e.g., governmental) review and approval processes, a or periods of public comment need to be carefully planned and weaved into the guideline timeline. 


\section{Collaboration between LSR and living guideline teams}

A smooth workflow between the team conducting the living guideline and the team producing the LSR should be ensured to maximize efficiency. That would require both coordinated, and ideally integrated, work processes between the two teams, and ideally integration of tools and platforms used for systematic reviewing and guideline development. This may raise some funding and logistical challenges.

\section{Thresholds for changing recommendations}

When engaged in a living guideline process, a living guideline panel might want to be prudent in changing the recommendations. First, and as discussed in the third paper of this series, (JCE XX) there might be an increased risk of false positive findings when a LSR leads to frequent updates of the meta-analyses. A false positive result may lead to inappropriate change in the direction and/or strength of the recommendation. This may in turn lead to harmful practices while potentially increasing resource use. A later correction of the false positive results leading to reverting the change in the recommendation, may negatively impact the trustworthiness of the guidelines. Second, frequently updated recommendations may pose significant challenges for dissemination and implementation.

On the other hand, the living guideline panel should not be too prudent. Setting the threshold to change a recommendation too high may diminish the potential benefits of living recommendations. Better understanding of how to establish these thresholds will be informed by methods to identify signals for the need to update systematic reviews, $(11,12)$ methods for repeated meta-analyses, (JCE, Simmonds et al) and the increasing experiences with living systematic reviews and living recommendations.

\section{Approaches to publication and dissemination}

A living recommendation can provide timely advice only if it is published and disseminated in a timely way. However, publishing and disseminating of individual recommendations (or cluster of recommendations) pose several challenges. One major challenge is the availability 
of a platform that allows publishing updates of individual recommendations (or cluster of recommendations). Another challenge is to ensure that users can clearly identify the latest version of the recommendation. At the same time, the guideline developer should provide access to the historical information related to the previous versions of the recommendation, including content information (i.e., the Evidence Profile and the EtD table) and process information (e.g., members of the guideline panel and their conflicts of interest declarations).

Another set of challenges, common to LSR and living guidelines, relates to authorship indexing and versioning.(JCE, Elliott et al.) For example, should authors who contributed to the original or earlier update remain authors on the latest one? Where guidelines are published in journal article form, should the updated recommendation be indexed with or separately from the original publication, and how should the citations of the update be allocated? Addressing these questions requires clear rules and agreements amongst authors, and between the guideline developer and the journal.

In terms of dissemination, digital online guideline documents (e.g., on websites, databases, or online textbooks) would allow any change in the recommendation to be reflected in realtime. However, this does not necessarily ensure that the target user is aware of this change. Potential solutions include a push mechanism, and linking living guidelines to information systems at the point of care, like electronic medical record and decision support tools. So, in an ideal world, as soon as a LSR triggers a change in a living recommendation, this change would be reflected in the online living guideline document, and in the linked downstream systems.

\section{Conclusion}

Living guidelines aim to provide timely advice for decision makers by optimizing the guideline development process to allow updating individual recommendations as soon as new relevant evidence becomes available. Guideline developers will need to prioritize the 
recommendations to switch the living status, according to explicit criteria. Implementing living guidelines will require both workflows to support them, and close collaboration between LSR and living guideline teams. Several challenges will face the implementation of living guidelines such as setting the thresholds for changing recommendations, and potential approaches to publication and dissemination. Implementing living guidelines will also bring logistical challenges to organizations, particularly when maintaining a relatively large number of living recommendations. Eventually, the success and sustainability of the concept of living guideline will depend on those of its major pillar, the LSR.

Guideline developers need to experiment with living guidelines while developing and refining the needed tools and processes. Efforts to build on include human and machine enablers of LSRs, (James et al. JCE xxx), formalizing guidelines in a computer-interpretable form,(13) and semantic technologies.(14) There is also a need to develop a research agenda to better understand the strengths and limitations of the approach, particularly in terms of feasibility, validity, cost, and efficiency relative to the standard approach. 


\section{Contributions}

1) conceived and designed the experiments: EAA, JJM

2) performed the experiments: N/A

3) analyzed and interpreted the data: N/A

4) contributed reagents, materials, analysis tools or data: N/A

5) wrote the paper: EAA, JJM, JE, LK, HJS

\section{Conflicts of interest declaration}

The authors declare no financial conflict of interest. They are all interested in the field of living guidelines and involved in projects to develop living guidelines.

\section{Acknowledgments}

The authors would like to thank the members of the Living Systematic Reviews network for their comments and suggestions on the paper. 


\section{References}

1. Graham R, Mancher M, Miller Wolman D, Greenfield S, Steinberg E. Clinical Practice Guidelines We Can Trust. Washington (DC)2011.

2. Andrews J, Guyatt G, Oxman AD, Alderson P, Dahm P, Falck-Ytter Y, et al. GRADE guidelines: 14. Going from evidence to recommendations: the significance and presentation of recommendations. J Clin Epidemiol. 2013;66(7):719-25.

3. Alonso-Coello P, Oxman AD, Moberg J, Brignardello-Petersen R, Akl EA, Davoli M, et al. GRADE Evidence to Decision (EtD) frameworks: a systematic and transparent approach to making well informed healthcare choices. 2: Clinical practice guidelines. BMJ. 2016;353:i2089.

4. Schunemann HJ, Wiercioch W, Etxeandia I, Falavigna M, Santesso N, Mustafa R, et al. Guidelines 2.0: systematic development of a comprehensive checklist for a successful guideline enterprise. CMAJ. 2014;186(3):E123-42.

5. Shojania KG, Sampson M, Ansari MT, Ji J, Doucette S, Moher D. How quickly do systematic reviews go out of date? A survival analysis. Ann Intern Med. 2007;147(4):224-33.

6. Martinez Garcia L, Sanabria AJ, Garcia Alvarez E, Trujillo-Martin MM, EtxeandiaIkobaltzeta I, Kotzeva A, et al. The validity of recommendations from clinical guidelines: a survival analysis. CMAJ. 2014;186(16):1211-9.

7. American College of Chest Physicians. Four Questions With Dr. Richard Irwin on CHEST Living Guidelines January 4, 2016 [Available from:

http://www.chestnet.org/News/Blogs/CHEST-Thought-Leaders/2016/01/Dr-Richard-Irwin-onCHEST-Living-Guidelines.

8. Brain Trauma Foundation. Living Guidelines Update July 72016 [Available from: https://www.braintrauma.org/news/article/guidelines-update.

9. INSIGHT Research. Living Guideline Group: keeping the Autism Spectrum Guideline up to date Sep 9, 2016 [Available from: http://www.health.govt.nz/our-work/disability-

services/disability-projects/autism-spectrum-disorder-guideline/living-guideline-group-keepingasd-guideline-date.

10. Vernooij RW, Sanabria AJ, Solà I, Alonso-Coello P, García LM. Guidance for updating clinical practice guidelines: a systematic review of methodological handbooks. Implementation Science. 2014;9(1):3.

11. Chung M, Newberry SJ, Ansari MT, Yu WW, Wu H, Lee J, et al. Two methods provide similar signals for the need to update systematic reviews. J Clin Epidemiol. 2012;65(6):660-8. 12. Shekelle P, Newberry S, Maglione M, Shanman R, Johnsen B, Carter J, et al. Assessment of the Need to Update Comparative Effectiveness Reviews: Report of an Initial Rapid Program Assessment (2005-2009). AHRQ Methods for Effective Health Care. Rockville (MD)2009.

13. Kaiser K, Miksch S. Versioning computer-interpretable guidelines: semi-automatic modeling of 'Living Guidelines' using an information extraction method. Artificial intelligence in medicine. 2009;46(1):55-66.

14. Slaughter L, Berntsen CF, Brandt L, Mavergames C. Enabling living systematic reviews and clinical guidelines through semantic technologies. D-Lib Magazine. 2015;21(1/2). 